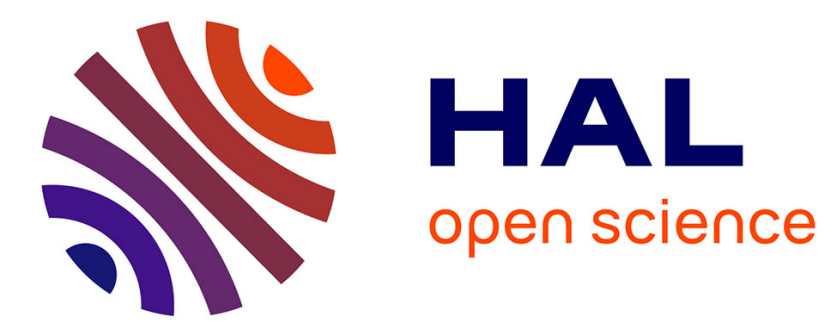

\title{
Un modèle efficace pour prédire le champ transitoire rayonné dans un milieu élastique par les traducteurs ultrasonores
}

\author{
A. Lhémery
}

\section{- To cite this version:}

A. Lhémery. Un modèle efficace pour prédire le champ transitoire rayonné dans un milieu élastique par les traducteurs ultrasonores. Journal de Physique IV Proceedings, 1994, 04 (C5), pp.C5-315-C5-318. 10.1051/jp4:1994564 . jpa-00253059

HAL Id: jpa-00253059

https://hal.science/jpa-00253059

Submitted on 1 Jan 1994

HAL is a multi-disciplinary open access archive for the deposit and dissemination of scientific research documents, whether they are published or not. The documents may come from teaching and research institutions in France or abroad, or from public or private research centers.
L'archive ouverte pluridisciplinaire $\mathbf{H A L}$, est destinée au dépôt et à la diffusion de documents scientifiques de niveau recherche, publiés ou non, émanant des établissements d'enseignement et de recherche français ou étrangers, des laboratoires publics ou privés. 


\title{
Un modèle efficace pour prédire le champ transitoire rayonné dans un milieu élastique par les traducteurs ultrasonores
}

\author{
A. LHÉMERY \\ Ecole Centrale de Paris, Laboratoire de Mécanique, URA 850 du CNRS, Equipe Ultrasons, Grande Voie \\ des Vignes, 92295 Châtenay-Malabry cedex, France
}

\begin{abstract}
A model is proposed for the transient radiation by an arbitrary loading in an elastic half-space. The assumptions and approximations made in the derivation are explicitely presented. Their consequences on model's accuracy are discussed. A radiation integral for elastic waves is derived and shown to be related to the Rayleigh integral for acoustic waves. As an example, analytic expression for the field radiated by a disc thickness-mode transducer is given, leading to a solution to displacement impulse-responses closely related to the well-known solution to transient acoustic radiation. Results predicted by the present model are compared with exact results. The accuracy is very satisfactory both qualitatively (shape of waveforms) and quantitatively (amplitude).
\end{abstract}

\section{INTRODUCTION.}

Pour simuler l'échographie ultrasonore large bande du contrôle non-destructif (CND), un modèle acoustique (les conversions de modes ne sont pas prises en compte) a été proposé [1] et simplifié [2]. L'importance des effets de diffraction par le traducteur dans la formation des échos a été montrée. Le champ transitoire rayonné (par réciprocité, reçu) est calculé analytiquement pour des traducteurs typiques de CND. Pour étendre le modèle aux ondes élastiques, il faut une solution pour le champ transitoire rayonné dans un solide. Avec le succès des méthodes impulsionnelles suite à l'article de Stepanishen [3] pour le calcul de l'intégrale de Rayleigh, des auteurs ont cherché à étendre le calcul aux ondes élastiques. Pour autant, le premier calcul exact et complet du déplacement dû à une source circulaire de traction normale a été donné par Eason en 1966 [4]. Laturelle [5] a obtenu par les mêmes transformations intégrales le champ transitoire de contrainte. D'autres transformations ont été proposées menant aussi à une solution exacte [6-9]. Qu'elle que soit la méthode utilisée, les résultats sont très complexes et demandent beaucoup de temps de calcul pour prédire une seule composante du déplacement en un point du champ. Deux solutions approximatives ont été proposées. Celle de Weight [10] consiste à pondérer la solution exacte acoustique [3] par des directivités empiriques des ondes élastiques. Schmerr et Sédov [11] obtiennent une solution analytique par développement asymptotique pour les points situés très près ou très loin de l'axe acoustique. Dans ces deux articles, les ondes de tête et de Rayleigh sont négligées. Des méthodes purement numériques ont aussi été utilisées pour ce calcul (éléments finis [12] différences finies [13]). Ces méthodes sont limitées en bande passante et ne permettent pas le calcul de réponses impulsionnelles. Il résulte de l'étude bibliographique que les méthodes menant à des résultats exacts sont trop lourdes numériquement pour être incluses dans un modèle général émission / diffusion / réception, et ne s'appliquent souvent qu'au seul cas du traducteur plan en contact en mode d'épaisseur. Les modèles approchés sont d'une grande calculabilité mais ne s'appliquent qu'aux traducteurs en contact. Dans la pratique du CND, le contrôle en immersion est très utilisé et ne trouve pas de solution parmi ces références. Dans la suite, nous proposons un modèle approximatif de très grande calculabilité (similaire à celle de l'intégrale de Rayleigh) permettant le calcul du champ rayonné par une source quelconque dans un milieu solide.

\section{THÉORIE.}

\subsection{Conditions initiales et aux limites}

Un chargement dynamique d'une pièce est modélisé par une source de traction à sa surface. On fait cinq hypothèses initiales. L'interface (notée $I T$ ) où la traction est appliquée est supposée plane. Une interface courbe peut être considérée si la diffraction secondaire est négligeable. Cette condition s'applique prati- 
quement à toutes les situations du CND. La pièce est supposée assez grande dans toutes les directions pour que le champ rayonné ne soit pas perturbé par la réflexion sur les limites de la pièce. On ne pourra pas modéliser le contrôle de plaques de faible épaisseur. On modélise ainsi la pièce par un demi-espace infini. La troisième hypothèse consiste à négliger les effets de couplage à l'interface $\mathrm{P}$. $\Pi$ est une surface libre en dehors de la partie $R$ où la traction est appliquée. Cette hypothèse est justifiée dans le cas de traducteur en contact mais ne l'est plus dans le cas d'un contrôle en immersion. Le couplage influence la propagation des ondes de surface mais pas des ondes de volume [14]. Parmi les méthodes de contrôle en immersion, seules celles utilisant les ondes de volumes seront modélisées correctement. La quatrième hypothèse consiste à limiter l'étude aux solides isotropes. Enfin, les champs de traction et de déplacement sont supposés initialement nuls. On décompose la frontière du demi-espace $V$ comme le montre Fig. 1. $V$ a pour densité $\rho_{0}$ et constantes élastiques (Lamé) $\lambda$ et $\mu$. Une partie de $\partial V, \Sigma$, est rejetée à l'infini où l'on impose au déplacement et à la contrainte de s'annuler (Sommerfeld pour les ondes élastiques [16]). $\Pi-R$ est une surface libre et la source de traction est imposée en $R$. On note $z$ l'axe normal à l'interface $\Pi$.

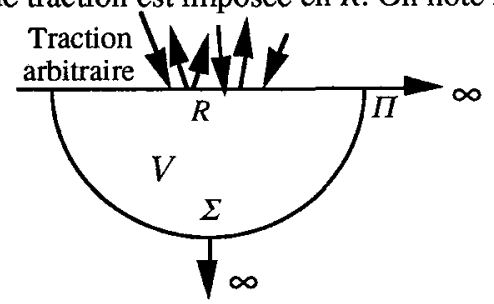

Fig. 1 - Décomposition du contour $\partial V$ du demi-espace élastique $V: \partial V=(\Pi-R)+R+\Sigma$.

\section{2. Équation intégrale du déplacement}

En un point $\mathbf{r}$ de $V$, le déplacement $\mathbf{u}$ est constitué de contributions dues à la contrainte et au déplacement lui-même en $\partial V$. Le déplacement peut être quelconque à l'interface $\Pi$ mais est nul en $\Sigma$. La contrainte est nulle partout en $\partial V$ sauf au niveau de la source en $R$. Partant de la formulation intégrale générale pour le déplacement (voir par exemple [15]) et en appliquant les conditions limites ci-dessus, on obtient,

$$
u_{n}(\mathbf{r}, t)=\int_{-\infty}^{\infty} d \tau \iint_{R} G_{i n}(\xi, t-\tau, \mathbf{r}, 0) T_{i}(\xi, \tau) d S(\xi)-\int_{-\infty}^{\infty} d \tau \iint_{\Pi} u_{i}(\xi, \tau) c_{i z k l}(\xi) G_{k n, l}(\xi, t-\tau, \mathbf{r}, 0) d S(\xi)
$$

où $i, k, l, n=x, y$ ou $z$. Le tenseur de Green $G_{i j}$ est la solution élémentaire du déplacement pour une force appliquée en $\xi$ dans la direction $x_{j}$ de dépendance temporelle $\delta(\tau)$ dans un espace infini [15],

$$
G_{i j}(\xi, \tau, \mathbf{r}, t)=\frac{\left(3 \gamma_{i} \gamma_{j}-\delta_{i j}\right)}{4 \pi \rho_{0}} \frac{1}{r} \int_{r / c_{L}}^{r / c_{T}} \tau \delta(t \tau) d \tau^{\prime}+\frac{\gamma_{i} \gamma_{j}}{\rho_{0} c_{L}^{2}} \frac{\delta\left(t \tau-\gamma / c_{L}\right)}{4 \pi r}-\frac{\gamma_{i} \gamma_{j}-\delta_{i j}}{\rho_{0} c_{T}^{2}} \frac{\delta\left(t-\tau-r / c_{T}\right)}{4 \pi r}
$$

où $\gamma_{i}=\left(\mathbf{r}-\mathbf{r}_{R}\right)_{j} / r, r=\left|\mathbf{r}-\mathbf{r}_{R}\right|$ et $\mathbf{r}_{R}$ point courant de $R . c_{L}$ et $c_{T}$ sont les vitesses des ondes de compression et de cisaillement. L'Eq. (1) est implicite donc complexe à calculer.

\subsection{Tenseur de Green simplifié - Intégrale explicite de rayonnement}

Les deux derniers termes de (2) correspondent à des ondes sphériques (en $r^{-1}$ ) de pure compression ou de pur cisaillement affectées de directivités. Le premier terme n'est ni irrotationnel, ni solénoïdal. Il varie comme $r^{-2}$ et est appelé terme de champ proche. Notre approximation consiste à le négliger. Soit $g_{i j}$ le nouveau tenseur, on peut suivre la démonstration connue pour l'obtention de l'intégrale de Rayleigh en faisant le choix de la "fonction miroir". $\xi$ ' étant l'image de $\xi$ par rapport à $\Pi$, on définit $\Gamma_{i j}$ par,

$$
\Gamma_{i j}(\xi, \tau ; \mathbf{r}, t) \equiv g_{i j}(\xi, \tau ; \mathbf{r}, t)+g_{i j}(\xi ;, \tau ; \mathbf{r}, t) .
$$

En $\Pi, \xi^{\prime}=\xi$ et $\Gamma_{i j}=2 g_{i j}$. La plus intéressante propriété de $\Gamma_{i j}$ est que son gradient est nul à l'interface. Dès lors, la deuxiéme intégrale (implicite) de l'Eq. (1) est nulle. Le seul terme restant est explicite. Du fait de la présence de la distribution $\delta(t)$, l'intégration temporelle est triviale. On obtient,

$$
u_{i}(\mathbf{r}, t)=\iint_{R}\left[\frac{\gamma_{i} \gamma_{j}}{\lambda+2 \mu} \frac{T_{j}\left(\mathbf{r}_{R}, t-\left|\mathbf{r}-\mathbf{r}_{R}\right| / c_{L}\right)}{2 \pi\left|\mathbf{r}-\mathbf{r}_{R}\right|}-\frac{\gamma_{i} \gamma_{j} \delta_{i j}}{\mu} \frac{T_{j}\left(\mathbf{r}_{R}, t-\left|\mathbf{r}-\mathbf{r}_{R}\right| / c_{T}\right)}{2 \pi\left|\mathbf{r}-\mathbf{r}_{R}\right|}\right] d S_{R}
$$


Cette intégrale ressemble à l'intégrale de Rayleigh pour le potentiel acoustique rayonné par une source de vitesse particulaire. Si cette dernière est exacte, celle donnée par (4) ne l'est pas (approximation champ lointain). Les deux termes n'expriment que les champs d'onde de compression et de cisaillement purs. Les autres ondes (de surface, de tête) ne peuvent pas être prédites par cette formulation. A partir de l'Eq. (4) et des relations contrainte/déformation, on peut calculer le champ de contrainte [17]. Pour évaluer l'influence de l'approximation sur la précision du modèle, on compare les résultats qu'il prédit avec des résultats exacts publiés pour le traducteur en contact fonctionnant en mode d'épaisseur.

\section{APPLICATION AU TRADUCTEUR DISQUE EN CONTACT EN MODE D'ÉPAISSEUR. \\ 3.1. Calcul de la réponse impulsionnelle du vecteur déplacement}

Un traducteur disque (de rayon $a$ ) plan en contact en mode d'épaisseur crée une traction $\mathbf{T}$ donnée par

$$
T_{z}\left(\mathbf{r}_{R}, t\right)=-p_{0} T(t), \text { si }\left|\mathbf{r}_{R}\right| \leq a,
$$

0 sinon, où $T(t)$ est la dépendance temporelle de la source. Du fait de la symétrie cylindrique, la composante tangentielle $u_{\theta}$ est nulle. Pour les composantes axiale $u_{z}$ et radiale $u_{r}$, la substitution de (5) dans (4) peut s'écrire sous la forme de produits de convolution. Pour chaque composante $i$, il y a un terme de compression $h^{i}{ }_{L}$ et un de cisaillement $h^{i} T$, i.e., $u_{i}(\mathbf{r}, t)=-p_{0} T(t) *\left[h^{i} L(\mathbf{r}, t)+h^{i} T(\mathbf{r}, t)\right]$ avec,

$$
h_{L}^{i}(\mathbf{r}, t)=\iint_{R}\left[\frac{1}{\lambda+2 \mu} \gamma_{i} \gamma_{z}-\frac{\delta\left(t-r / c_{L}\right)}{2 \pi r}\right] d S_{R} \text { et } h_{T}^{i}(\mathbf{r}, t)=-\iint_{R}\left[\frac{1}{\mu}\left(\gamma_{i} \gamma_{z}-\delta_{i z}\right) \frac{\delta\left(t-r / c_{T}\right)}{2 \pi r}\right] d S_{R} \text {. }
$$

Pour calculer les différentes réponses impulsionnelles de déplacement [17], on a suivi exactement le calcul du potentiel impulsionnel acoustique [3]. Le calcul fait intervenir le changement de variable $d S_{R} / r=$ $2 c d t d \varphi$. Les cosinus directeurs s'expriment par $\gamma_{r}=\left[c^{2} t^{2}-z^{2}\right]^{1 / 2} \cos \varphi / c t$ et $\gamma_{z}=z / c t$. Soit $\varphi_{\text {lim }}$ l'angle dont les valeurs sont données dans le tableau İ, les réponses impulsionnelles sont données par,

$$
\begin{array}{ll}
h_{L}^{z}(\mathbf{r}, t)=\frac{1}{\rho_{0} c_{L}} \frac{z^{2}}{c_{L}^{2} t^{2}} \frac{\varphi_{\text {lim }}\left(c_{L}\right)}{\pi}, & h_{L}^{r}(\mathbf{r}, t)=\frac{1}{\rho_{0} c_{L}} \frac{z \sqrt{c_{L}^{2} t^{2}-z^{2}}}{c_{L}^{2} t^{2}} \frac{\sin \left[\varphi_{\text {lim }}\left(c_{L}\right)\right]}{\pi}, \text { pour } t_{0}^{L} \leq t \leq t_{2}^{L}, \\
h_{T}^{z}(\mathbf{r}, t)=\frac{1}{\rho_{0} c_{T}} \frac{c_{T}^{2} t^{2}-z^{2}}{c_{T}^{2} t^{2}} \frac{\varphi_{\text {lim }}\left(c_{T}\right)}{\pi}, & h_{T}^{r}(\mathbf{r}, t)=-\frac{1}{\rho_{0} c_{T}} \frac{z \sqrt{c_{T}^{2} t^{2}-z^{2}}}{c_{T}^{2} t^{2}} \frac{\sin \left[\varphi_{\text {lim }}\left(c_{T}\right)\right]}{\pi}, \text { pour } t_{0}^{T} \leq t \leq t_{2}^{T},
\end{array}
$$

avec $t_{0}=z / c, t_{1}=\left[z^{2}+(a-\rho)^{2}\right]^{1 / 2} / c$ et $t_{2}=\left[z^{2}+(a+\rho)^{2}\right]^{1 / 2} / c$, avec $c=c_{L}$ ou $c_{T}$. Avec les mêmes notations, le potentiel impulsionnel acoustique est donné par $h(\mathbf{r}, t)=c \varphi_{\text {lim }} / \pi$, pour $t_{0} \leq t \leq t_{2}$.

Tableau I - Valeur de $\varphi_{\text {lim }}$, fonction de $\rho, z$ et $c t . A=c^{2} t^{2}-z^{2}+\rho^{2}-a^{2}, B=2 \rho\left[c^{2} t^{2}-z^{2}\right]^{1 / 2}, C=\left[c^{2} t^{2}-z^{2}\right]^{1 / 2} / 2 a$.

\begin{tabular}{lcccc}
\hline \hline$\varphi_{\text {lim }}$ & $\rho=0$ & $0<\rho<a$ & $\rho=a$ & $\rho>a$ \\
\hline$t<t_{0}$ ou $t>t_{2}$ & 0 & 0 & 0 & 0 \\
$t_{0} \leq t \leq t_{1}$ & $\pi$ & $\pi$ & $\pi / 2 \quad\left(t_{0}=t_{1}\right)$ & 0 \\
$t_{1} \leq t \leq t_{2}$ & 0 & $\cos ^{-1}[A / B]$ & $\cos ^{-1}[C]$ & $\cos ^{-1}[A / B]$ \\
\hline
\end{tabular}

\subsection{Comparaison avec des résultats exacts}

La solution développée en 3.1. permet une comparaison directe avec les résultats exacts [18]. Dans [18], les résultats concernent des vitesses particulaires et non des déplacements. Il suffít de dériver temporellement les résultats du déplacement. Nous avons comparé systématiquement les résultats donnés en [18] avec nos propres prédictions [17]. Nous ne montrons ici que quelques exemples. Le traducteur est un disque $20 \mathrm{~mm}-\emptyset$ vibrant uniformément ou un traducteur annulaire ( $\emptyset$ interne: $16 \mathrm{~mm}$, externe $20 \mathrm{~mm}$ ). Pour ce dernier, on soustrait les résultats d'un traducteur de $16 \mathrm{~mm}$ de ceux d'un de $20 \mathrm{~mm}$. La dépendance temporelle est un cycle de sinusö̈de à $5 \mathrm{MHz}$ (fréquence centrale $v_{c} \approx 4.4 \mathrm{MHz}$ ). Les résultats publiés étaient pour une distance de $15 \mathrm{~mm}$ du traducteur. Selon la théorie monochromatique, le champ lointain pour les ondes $L$ commence à $z_{l i m}=a^{2} v_{C} / c_{L} \approx 75 \mathrm{~mm}$. La distance $15 \mathrm{~mm}$ est donc caractéristique du champ proche. Notre approximation "champ lointain" risque d'être prise en défaut. Pourtant, les figures suivantes montrent un très bon accord entre les résultats exacts ou calculé par Eqs. (7), tant qualitatif (forme d'onde, instants caractéristiques) que quantitatif (les résultats sont représentés à la même échelle que dans [18]). Les contributions d'ondes de tête (indiqués par $H W$ ) dans les résultats exacts ne sont pas prédits par notre calcul. Il y a un artefact dans nos résultats pour les vitesses axiales, arrivant à $t_{0}^{T}$. Il est à $24.4 \mathrm{~dB}$ en dessous des contributions principales et n'est donc pas trop problématique. 
Calculs exacts [18] Calculés avec Eq. (7)
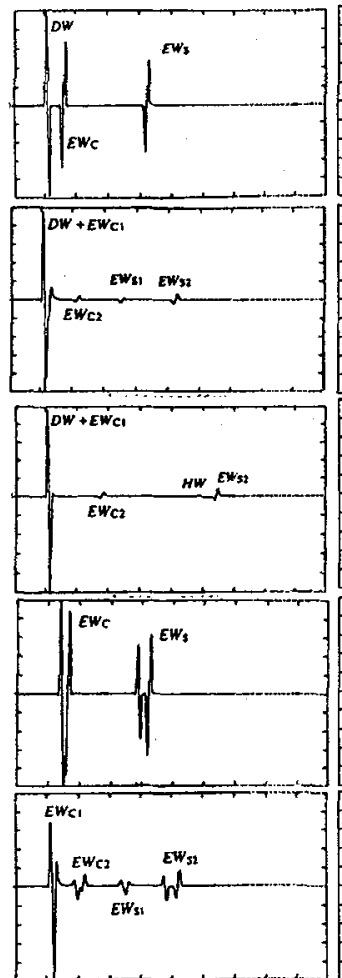
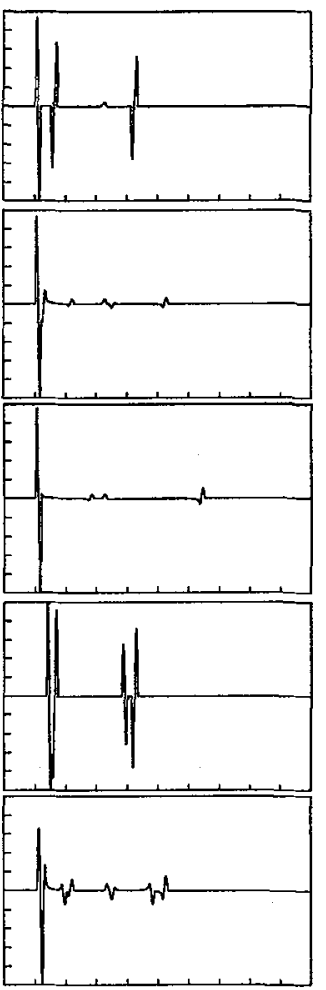

Calculs exacts [18] Calculés avec Eq. (7)

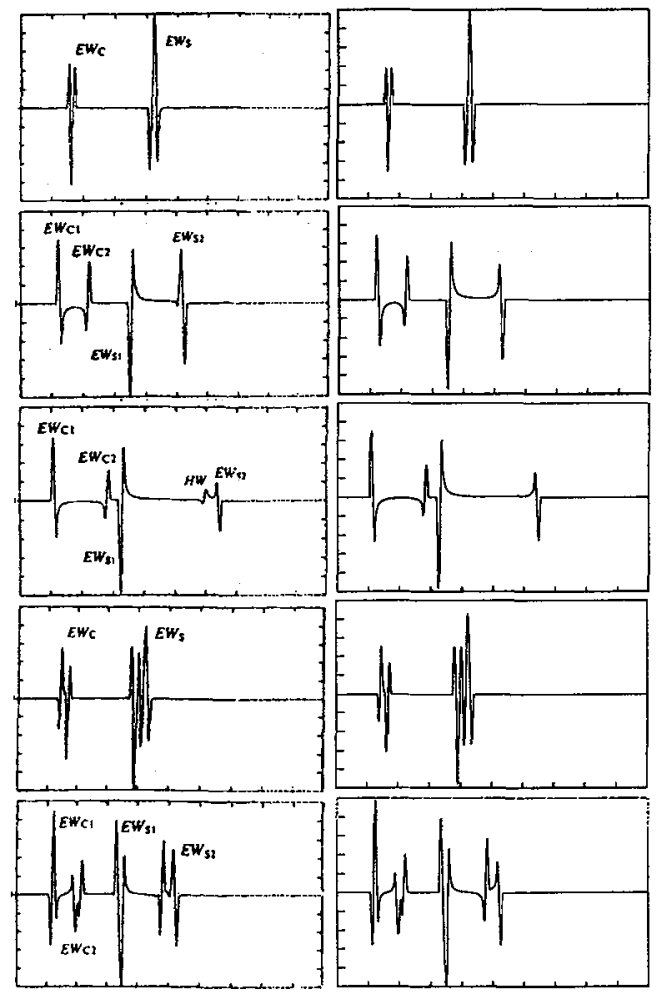

Fig. 2 - Colonnes 1, 2: $v_{z}$, colonnes 3, 4: $v_{r}$. Lignes 1, 4: $\rho=0\left(v_{z}\right), \rho=0.5\left(v_{r}\right)$, lignes 2, 5: $\rho=5$, ligne 3: $\rho=10 \mathrm{~mm}$. Lignes $1,2,3$ : traducteur circulaire, lignes 4,5 : traducteur annulaire. (1 $\mu \mathrm{s} / \mathrm{div}$.)

\section{CONCLUSION.}

La formule intégrale approximative proposée permet de calculer le champ rayonné par une source ultrasonore quelconque dans un solide. Elle est très proche de l'intégrale exacte de Rayleigh pour l'émission dans les fluides. On peut profiter de cette similitude pour appliquer les calculs développées en acoustique aux ondes élastiques. L'exemple donné (piston circulaire) illustre cette possibilité. Les résultats obtenus confirment le modèle empirique de Weight [10].

[1] Lhémery A., J. Acoust. Soc. Am. 90 (1991) 2799-2807.

[2] Raillon R., Thèse de doctorat de l'École Centrale Paris (1993).

[3] Stepanishen P.R., J. Acoust. Soc. Am. 49 (1971) 1629-1638.

[4] Eason G., J. Inst. Maths. Applics. 2 (1966) 299-326.

[5] Laturelle F.G., Wave Motion 12 (1990) 107-127.

[6] Kawashima K., I.E.E.E. Trans. Son. Ultrason. SU-31 (1984) 83-94.

[7] Bresse L.F. and Hutchins D.A., J. Acoust. Soc. Am. 86 (1989) 810-817.

[8] McNab A., Cochran A. and Campbell M.A., J. Acoust. Soc. Am. 8 (1990) 1455-1465.

[9] Djelouah H. and Baboux J.-C., J. Acoust. Soc. Am. 92 (1992) 2932-2941.

[10] Weight J.P., J. Acoust. Soc. Am. 81 (1987) 815-826.

[11] Schmerr Jr. L.W. and Sedov A., J. Acoust. Soc. Am. 86 (1989) 1988-1999.

[12] Laturelle F.G., Computers and Structures 32 (1989) 721-735.

[13] Ilan A. and Weight J.P., J. Acoust. Soc. Am. 88 (1990) 1142-1151.

[14] Roberts R.A., Wave Motion 12 (1990) 583-593.

[15] Aki K. and Richard P.G., Quantitative seismology - Theory and experiments (W.H. Freeman and Company, San Francisco, 1980), Ch. 2 - 3.

[16] Pao Y.H. and Varatharajulu V., J. Acoust. Soc. Am. 59 (1976) 1361-1371.

[17] Lhémery A., soumis à publication dans J. Acoust. Soc. Am.

[18] Baboux J.-C. and Kazys R., J. Acoust. Soc. Am. 92 (1992) 2942-2951. 